Research Article

\title{
Applied Research of Knowledge in the Field of Artificial Intelligence in the Intelligent Retrieval of Teaching Resources
}

\author{
XuJing Bai $\mathbb{D}$ and JiaJun Li \\ School of Management, Northwestern Polytechnical University, Xi'an 710072, China \\ Correspondence should be addressed to XuJing Bai; bxjblue@mail.nwpu.edu.cn
}

Received 1 April 2021; Revised 20 May 2021; Accepted 27 May 2021; Published 14 June 2021

Academic Editor: Chenxi Huang

Copyright ( $\odot 2021 \mathrm{XuJing}$ Bai and JiaJun Li. This is an open access article distributed under the Creative Commons Attribution License, which permits unrestricted use, distribution, and reproduction in any medium, provided the original work is properly cited.

\begin{abstract}
In the development process of education informatization, digital teaching resources continue to grow, and how to manage and organize massive teaching resources has become a key issue for teaching staff. An efficient and accurate search system is an important part of the teaching resource service system. The use of intelligent search engines can search teaching resources comprehensively and efficiently, and the artificial intelligence search engine provides a reliable and convenient solution for the design and development of intelligent search systems. The article analyzes the design principles and technical standards of the intelligent search system, expounds the system's functional architecture and database design, and introduces the realization process and principles of the search system. By analyzing the characteristics of basic education resources and existing automatic abstracting methods, this paper proposes to integrate the calculated feature word weights in the field of basic education into the algorithm for calculating the weights of abstract sentences and simultaneously examine the sentence position, sentence length, and other texts. There is an automatic summarization algorithm for surface statistics. This article also introduces the search design ideas and implementation steps based on artificial intelligence, makes a scientific evaluation and summary of the actual situation of the automatic abstract system running in the basic education resource search engine, and looks forward to the next improvement work.
\end{abstract}

\section{Introduction}

At present, all kinds of schools in our country attach great importance to the construction of teaching resource databases and course databases. They have independently developed a large number of teaching resource databases such as courseware resource databases, course resource databases, and resource websites [1]. Teachers and students often use these resource databases to obtain learning resources and participate in the Internet. Teaching activities can effectively improve the information collection and processing capabilities of teachers and students. However, there are still the following problems in the process of using: a large number of repeated constructions of teaching resources and the established teaching courseware, electronic teaching plans, and course videos are insufficient in classification and resource organization standardization and perfection. The results obtained by learners are often similar or even the same information when searching. It takes a lot of time to perform manual secondary retrieval and filtering; learners have individualized needs for the acquisition of the expression of teaching resources [2]. It is necessary to quickly find the target resource through positioning and retrieval. However, the resource organization structure of various teaching resource libraries and resources is different or heterogeneous, the resource manifestation is single, and the storage format is diverse [3]. The manifestation of the acquired teaching resources cannot meet the needs of learners and makes the acquisition of teaching resources more difficult.

This paper analyzes the relevant knowledge and technology of search engines, especially after analyzing some limitations of search engines, and puts forward an intelligent search engine model suitable for students in the environment of teaching 
resources. The architecture of the intelligent search engine is given. In this model, the semantic network and intelligent agent technology in artificial intelligence technology are introduced to improve the intelligence of search engines. According to the characteristics of teaching resources, based on the classification of educational disciplines, the concept of a semantic network is constructed to realize the expansion of students' query requests, including synonymous expansion, related associations, and conceptual expansion. In order to achieve the purpose of expanding the scope of retrieval and improving the recall rate of search engines, by analyzing the feedback information of students, a personal interest model of students is established on the structure of the conceptual semantic network, and the degree of relevance of the concepts is expressed through the connection between the concepts. The model can intuitively reflect the range of students' personal interests. By establishing the range of students' interests, the retrieved documents will be reordered, so as to improve the ranking of the documents that students are interested in and improve the accuracy of search engines. This paper also tested and verified the intelligent agent system and gave the test results. The results show that the intelligent agent system can effectively improve the ranking of related documents and provide students with educational resources that interest them.

This article mainly describes the content of the paper from five parts. The specific arrangements of each chapter are summarized as follows. The first section is the introduction, which mainly introduces the specific topic background and research significance of the paper and analyzes the artificial intelligence search model and educational resource retrieval. The status quo summarizes the main research content and the structure of the paper. The second section discusses related work. The third section introduces the application of artificial intelligence technology in the intelligent retrieval of teaching resources. The fourth section analyzes the design of educational resource search of the artificial intelligence search algorithm, and the fifth section verifies the performance of artificial intelligence search through experimental simulation. The sixth section summarizes and looks forward to this article.

\section{Related Work}

With the continuous growth of information on the Internet, people have become a way of learning to find what they need through the Internet [4]. In particular, for students in education, their learning is based on resource learning. Therefore, obtaining useful subject knowledge is a key issue for online education students to improve their knowledge. Artificial intelligence search has gradually become a research hotspot for scholars at home and abroad [5]. The application of artificial intelligence search knowledge has received extensive attention and development in recent years. The research on artificial intelligence search knowledge in intelligent search of teaching resources is as follows.

Related scholars have conducted research on the on-site search subsystem and Chinese intelligent search engine in the online teaching platform. The guiding ideology of online teaching activities is a learner-centered educational philosophy, which aims to improve their own learning ability through learners' independent learning and collaborative learning. The emergence and use of online teaching platforms provide a platform for learners and teachers to share teaching resources [6]. However, due to the massive number of teaching resources in the online teaching platform, the content and form of courses, after-school exercises, reference materials, teaching courseware, etc, they have the characteristics of diversity, which makes them difficult for teachers and learners to quickly search and apply resources in teaching activities [7]. How to maximize the sharing of teaching resources so that learners can quickly and accurately find the resources they need from their own resources is the main task of the development of search engines in the education field, and the development of a platform that can be applied to online teaching the resource search tool in is the core to solve this problem.

Related scholars analyzed the characteristics of online teaching platforms and the characteristics of online education, combined with the evaluation standards of online learners' learning ability, confirmed the theoretical value of search tools in online teaching platforms, and fully analyzed the working principles of search engines, and on the basis of key technologies, a search framework based on open source is designed. The overall architecture of the search system on the site, the introduction of information push technology and intelligent knowledge base technology into the framework, has improved the intelligence level of search engines, in addition, analyzed and compared the shortcomings of the word segmentation of existing search engines, proposed a word segmentation optimization plan, and optimized the search result ranking technology, making the search tool more suitable for learners to search and query massive resources in the teaching platform, and can quickly locate the location of the data required by the user [8]. The search results of the required resources are fed back to the users to further improve the search quality of the search engine. In the process of online teaching, learners mainly obtain the information resources they need through online media [9]. Whether learners can make full use of the teaching resources on the Internet is the main factor that affects their knowledge construction. However, for the current messy network in terms of teaching resources, learners will inevitably produce the phenomenon of "information trek". These factors will indirectly affect learners' interest and enthusiasm for learning. For this reason, using search engines to assist online teaching can improve resource utilization [10]. Search engines can not only improve the utilization of online teaching resources but also be one of the key technologies that need to be used in the development of online teaching.

\subsection{Application of Artificial Intelligence Technology in Intelligent Retrieval of Teaching Resources}

2.1.1. Construction of Artificial Intelligence Retrieval Ontology Model. Ontology is a clear and standardized description of conceptual models, while domain ontology is an abstract description of concepts and relationships in specific 
domains. Its functions are similar to relational models, and it is an organizational framework for information resources in related domains [11]. Domain ontology is the starting point of the entire intelligent information retrieval system, and it also runs through the entire system structure, provides references and basis for other functional modules, and plays a pivotal role in the entire system. The construction of domain ontology is a complex system engineering, which requires the participation of many domain experts and a lot of time investment [12]. Various ontology construction methods have been proposed. In order to ensure that a suitable domain ontology can be established, this paper fully draws on the ideas and experience of software engineering and proposes a new prototype ontology construction method based on the spiral model. The ontology construction process is shown in Figure 1.

\subsection{Application of Intelligent Classification Technology in the} Classification of Teaching Resources. There are many kinds of teaching resources and a large number of them. Most of the data and information contained in them are in disorder. These data, information materials, and knowledge are presented in different formats and languages, and the source of each information resource is also different. In order to find accurate information, data, and knowledge as soon as possible, it is necessary to do a good job of processing information in advance [13]. The use of intelligent classification technology in archive classification management can effectively manage the messy knowledge, information, and data in the intelligence of teaching resources. According to certain standards, the huge and disorderly knowledge, information, and data can be classified in time to facilitate the location and acquisition of information data [14]. When categorizing, you can first divide the knowledge, information, and data into several major topics according to the theme's standards and then divide each major topic into several different small topics to construct a hierarchical topic structure. Teaching resource intelligence can be intelligently classified with the support of artificial intelligence technology, pattern recognition, natural language processing, and machine learning. In the technology of natural language processing, the text classification technology is based on a practice text sample library, and this sample library has been marked. In this sample library, a model that is related to text types and text attributes is found, and then, the found relational model is used to distinguish new text categories. The intelligence of text-based teaching resources can be intelligently classified with the support of text classification technology [15]. In addition, archivists can also intelligently distinguish and classify the teaching resources of multimedia categories by intelligent classification technology composed of voice recognition, image recognition, and video recognition. The system structure of intelligent search is shown in Figure 2.

\subsection{Application of Intelligent Search Technology in Intelligent} Retrieval of Teaching Resources. With the continuous development of artificial intelligence technology, the types and quantity of teaching resource intelligence have grown rapidly, which has had a huge impact on traditional archival information resource retrieval [16]. The disadvantages of traditional retrieval methods are obvious, and retrieval efficiency has fallen far short of the requirements of archival work. Therefore, in order to improve the efficiency of archive retrieval, it is necessary to use intelligent retrieval technology to replace traditional archive retrieval methods. Intelligent retrieval makes full use of various artificial intelligence technologies such as pattern recognition and natural language processing [17]. The biggest difference between intelligent search and traditional search is as follows: intelligent search can search for the most similar results to the search content entered by the user and can also arrange these search results in the most similar order, which can greatly reduce the search time for users and improve retrieval efficiency. In addition to searching for text information, intelligent retrieval can also search for multimedia information such as sound, image, and video. In the current Internet age where multimedia teaching resources are more and more intelligent, intelligent retrieval technology has the advantages that traditional information retrieval technology cannot compare and has played a huge role in archive retrieval work [18]. The most fundamental purpose of file classification management is to maximize the use of information, data, and knowledge in the intelligence of teaching resources and to manage them efficiently. The intelligence, data, and knowledge of teaching resources have a wide variety and huge amount, and the sources of information, data, and knowledge are also different, which creates obstacles for users to search for the useful information [19]. Therefore, in order to meet the needs of users for archive search and want users to find the information they need in the shortest time, new search technologies must be introduced. Neural network technology is an artificial intelligence technology that imitates the behavioral characteristics of animal neural networks to perform distributed processing of information. In the file classification management work, the comprehensive introduction of neural network technology can greatly improve the searchability of search engines. As long as an intelligent search engine is developed, an accurate search of information, data, and knowledge in the intelligence of teaching resources can be realized, and the problem of information, data, and knowledge overload can be solved, so as to realize the effective management of archives classification [20]. The application of the intelligent search engine is based on neural network theory and statistics to quickly analyze massive information, and quickly classify it, and transmit the information and data related to the input search content to the user. In addition, the intelligent search engine can also automatically grasp the complex concepts in the new information data, automatically learn the meaning of the concepts, and then store the new concepts, which is convenient for searching and searching for the concepts in the future [2]. Adding neural network and pattern recognition to intelligent search will enable intelligent search to locate and search for information based on the text content, contextual connection, and meaning. Therefore, the 
intelligent search can quickly find the content which is the closest and most useful information, and it can also provide users with an expandable traditional system of information, data, and knowledge, thus greatly improving the utilization rate of teaching resource intelligence. The system framework of intelligent information retrieval is shown in Figure 3.

This model is mainly composed of two relatively independent parts: an offline processing part and an online processing part. The offline processing part is mainly responsible for the collection of original information, the establishment of domain ontology, and the processing of original information. The online part of the processing mainly includes obtaining the user's query request, query condition coding, querying the semantic index library through the search engine, sorting the retrieved result set, and returning the sorted final result to the user. The offline processing part is relatively time-consuming, so you can choose to disconnect the service and the server load is light.

\section{Design of Educational Resource Search Based on Artificial Intelligence Search Algorithm}

\subsection{Characteristics of Intelligent Search Engine in Teaching} Platform. Traditional search engines have many problems in function, such as a large amount of information feedback in search results and more irrelevant information. This is mainly because traditional search engines mainly use keyword-based mechanical matching methods to achieve users' search needs [21]. To a certain extent, the ability to understand and analyze search sentences is not accurate enough; that is to say, traditional search engines cannot perform fast and accurate searches for users' personalized knowledge and professional knowledge in different fields. However, with the further development of search engine technology, the emergence of smart search engines can solve the above problems [7]. Smart search engines can provide users with a friendly search interaction interface and can improve the original resource search based on keyword search to knowledge understanding. They can search for the most meaningful resources for users from existing resources, give feedback according to the user's search request, and can realize automatic word segmentation technology, synonym technology, machine learning technology, etc. Intelligent search engines will have more personalized features, can gradually understand users' search preferences based on information such as resource browsing habits and search habits of different users, establish corresponding user description model information for different users, and actively provide users with relevant information, to bring users a better search experience [22].

The in-site intelligent search engine framework proposed in this article is designed for domestic learners of the teaching platform. Through the investigation of the use of the teaching resource library in the online teaching platform, it is found that most of the resources in the resource library are documents, mainly including document resources such as teaching courseware and extracurricular reference materials in such formats. Therefore, the intelligent search system in the teaching platform should mainly have the following five characteristics:

(1) Intelligent and personalized search function: the intelligence of search engines is mainly reflected in the ability to obtain users' search preferences according to their search habits and then provides different users with different types of information. In this article, the search engine should be able to construct a learner's interest model according to the learner's characteristics and update this interest model in real time, so as to determine the scope of the search and provide its professional and personalized search service

(2) Facing the field of teaching resources and strong professionalism: at present, general-purpose commercial search engines cannot well meet the personalized search needs of users in a certain field. The in-site intelligent search engine in the teaching platform realizes the function of quickly searching for the teaching resources in the platform and obtains the corresponding teaching resources according to the theme, which shortens the time for learners to obtain resources

(3) Good user interface: when designing the user interface, you can refer to the current popular search engines, combine the functions of the online teaching platform itself, and add in-site links as appropriate. For example, you can specify which courses the teaching resources come from, and which specific teaching modules in the course, etc. improve the user's search experience.

(4) Providing learners with search recommendations: the on-site teaching resource search engine is a search engine oriented to a specific field. The search tool needs to provide learners with some more commonly used keyword input prompts related to teaching resources, use natural language to interact with learners, and adopt technologies such as semantic web and automatic word segmentation technology which accurately and quickly understands learners' search requests

(5) Higher search result accuracy rate: search engines use information filtering, information mining, and other technologies to filter the teaching resources in the network teaching platform and combine the search sentences to filter the importance of learners again, so that the feedback search results are more in line with the learners' search needs

\subsection{Artificial Intelligence Applied to Search Engine Resource} Acquisition. From the above analysis, we can know that the existing search engine resource acquisition methods are blind. Relying on width and depth-first algorithms, they often get a lot of irrelevant information, which leads to the decline of efficiency and search accuracy. So how can we improve the efficiency and accuracy of search and how can 


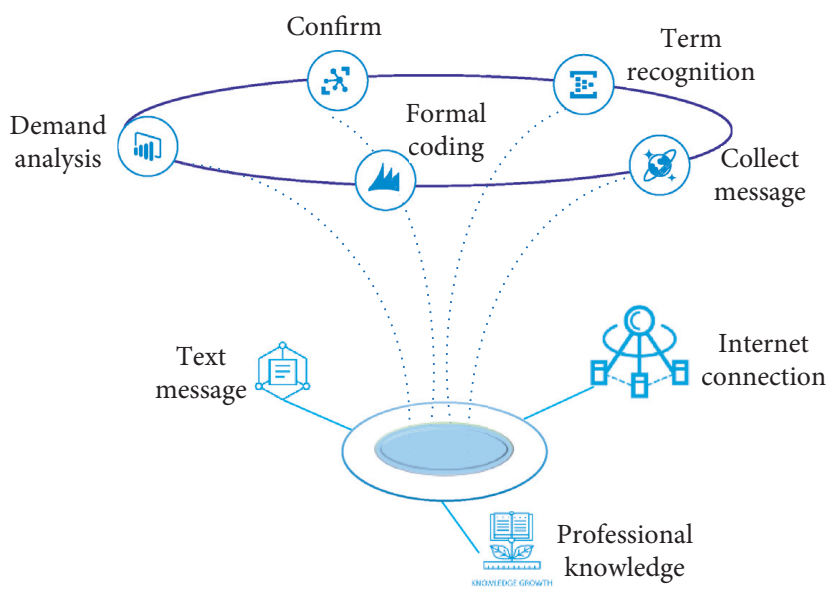

FiguRe 1: Prototype method of domain ontology construction.

we obtain more valuable information resources at the same time? If the required information is on the page and the depth-first search algorithm is used, then the previous must be traversed; it is the turn to visit the page after a page, the price paid is very large, but the efficiency is only about $10 \%$. If the heuristic search is used, generally speaking, it can be accessed as long as the page is traversed, which is much more efficient than the depth-first algorithm. The application of artificial intelligence to web crawlers will enable search engines to achieve greater success in the acquisition of information resources [23]. Using heuristic algorithms, web crawlers can eliminate irrelevant links when traversing links, and only visit and traverse links that are deemed useful after the evaluation function are calculated. This greatly reduces the number of traversed links, and the obtained pages that meet the requirements account for a large proportion of the entire traversed pages. The accuracy of the collection of information resources by web crawlers has also improved. Intelligent agents, also known as agents, are new results of artificial intelligence research. It can perform various complicated tasks on behalf of the user according to the user's needs without clear specific requirements. The application of intelligent agent technology to search engines will provide a broad space for the development of a new generation of more powerful online intelligent search systems. When accessing a web page link, how to judge the type of the page is a tricky problem. If you can develop an intelligent agent system, let it automatically complete the classification of documents or judge its type; it would be a better way. The main processing steps taken by the artificial intelligence search algorithm are shown in Figure 4.

The so-called network information retrieval also refers to Internet retrieval. With the function of network interface software, users can query information in various fields on the terminal. This retrieval method is mainly based on the distribution function of the Internet, that is, data distribution and storage, and a large amount of information is distributed in the corresponding server. The user searches for the dispersedly stored data through the application terminal system, and any data can be searched and applied on the network. The basic structure of the network information retrieval system is shown in Figure 5.

First, take the knowledge base as an example. As the part responsible for long-term memory in the artificial intelligence system, this part mainly stores specialized knowledge. This includes established facts and information, as well as general common sense and rules. Some special systems also contain databases. The second is the inference engine. As a set of programs for performing retrieval tasks, it mainly includes master control and various task programs. As a specialized library, it plays an important role in providing search support. The user interface mainly includes the system and the links used by the user to input and convey relevant information. As a bridge between external information and internalization, it can display the final processing result to the user and transfer the user's will to the computer. The nonnatural language used in this process can also reduce the burden and pressure of users in the process of use. The intermediate database is also the blackboard we often say; it is mainly used to store intermediate results and data in the process of performing tasks and inferences. In the actual application process, the system will first display the problem on the blackboard and then display the initial state of the problem. Then, the expert system makes inferences based on the knowledge search matching status of the knowledge base and, at the same time, constantly repairs and infers the contents of the blackboard. When necessary, we will also ask customers to make up for and solve the shortage of knowledge in the knowledge base. Therefore, the blackboard can also be regarded as a dynamic knowledge base to a certain extent. It plays a very critical role in controlling the progress of the job. Finally, the knowledge acquisition device is also called a learner. Its main function is to continuously repair the content of the knowledge base based on the experience of system operation. This is an effective supplement to ensure the smooth operation of the system. The interpreter is mainly responsible for solving the user's questions and is also responsible for explaining to the user the calculation trajectory of the system conclusion. In general, artificial intelligence technology is a means of science and 


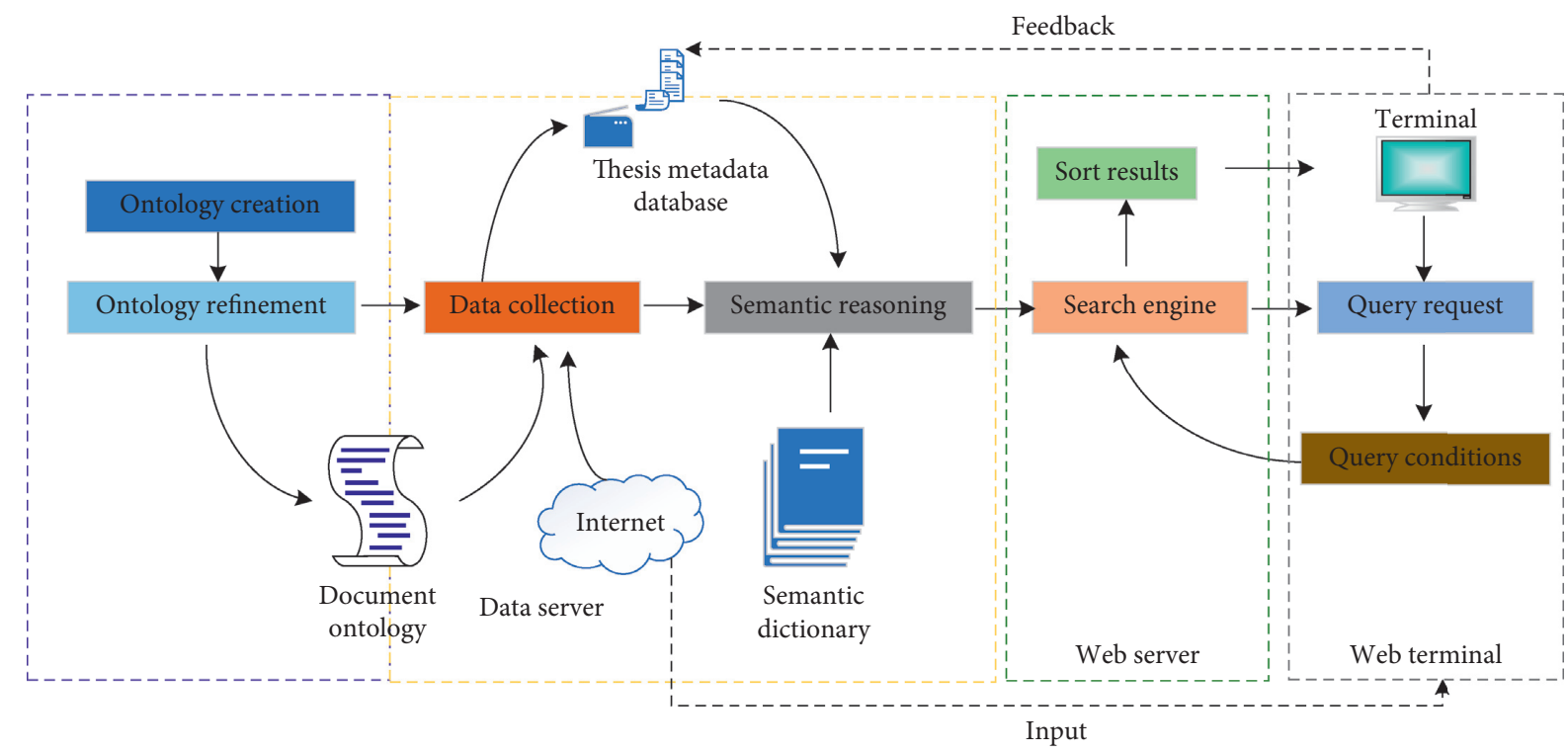

FIgURE 2: The system structure of intelligent search.

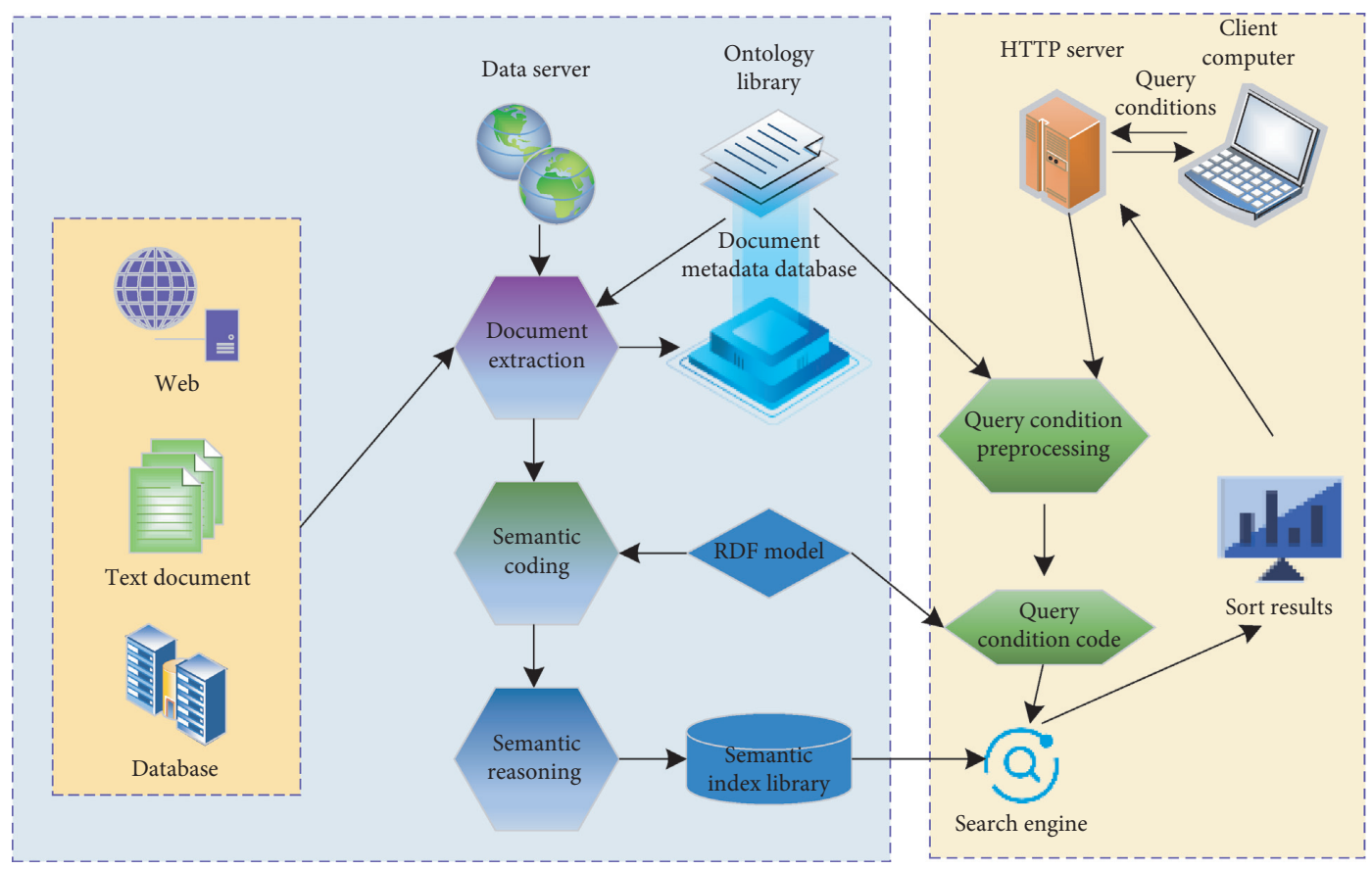

Figure 3: Framework for intelligent information retrieval.

technology to help people solve some urgent problems in the form of analogy thinking.

\section{Experimental Results and Analysis}

4.1. Data Set and Experiment Establishment. In order to objectively evaluate the abovementioned feature selection method in the actual situation of basic education text classification, we select basic education discipline resources to construct a hierarchical classification system with a twotier structure. As shown in Figure 6, there are 12 major categories and 18 subcategories and manually obtained a corresponding data set, which includes 8428 training documents and 1867 open test documents. The specific distribution is shown in Figure 6. In the experiments and discussions later in this article, unless otherwise specified, they refer to the training set and test set.

The classification algorithm used in the experiment is the Roccio algorithm. In the actual application, $\alpha=1$ and $\beta=0$ in the Roccio formula, that is, only positive samples are considered, and the category vector is the center of the training text of the category, because in many cases, the effect of 


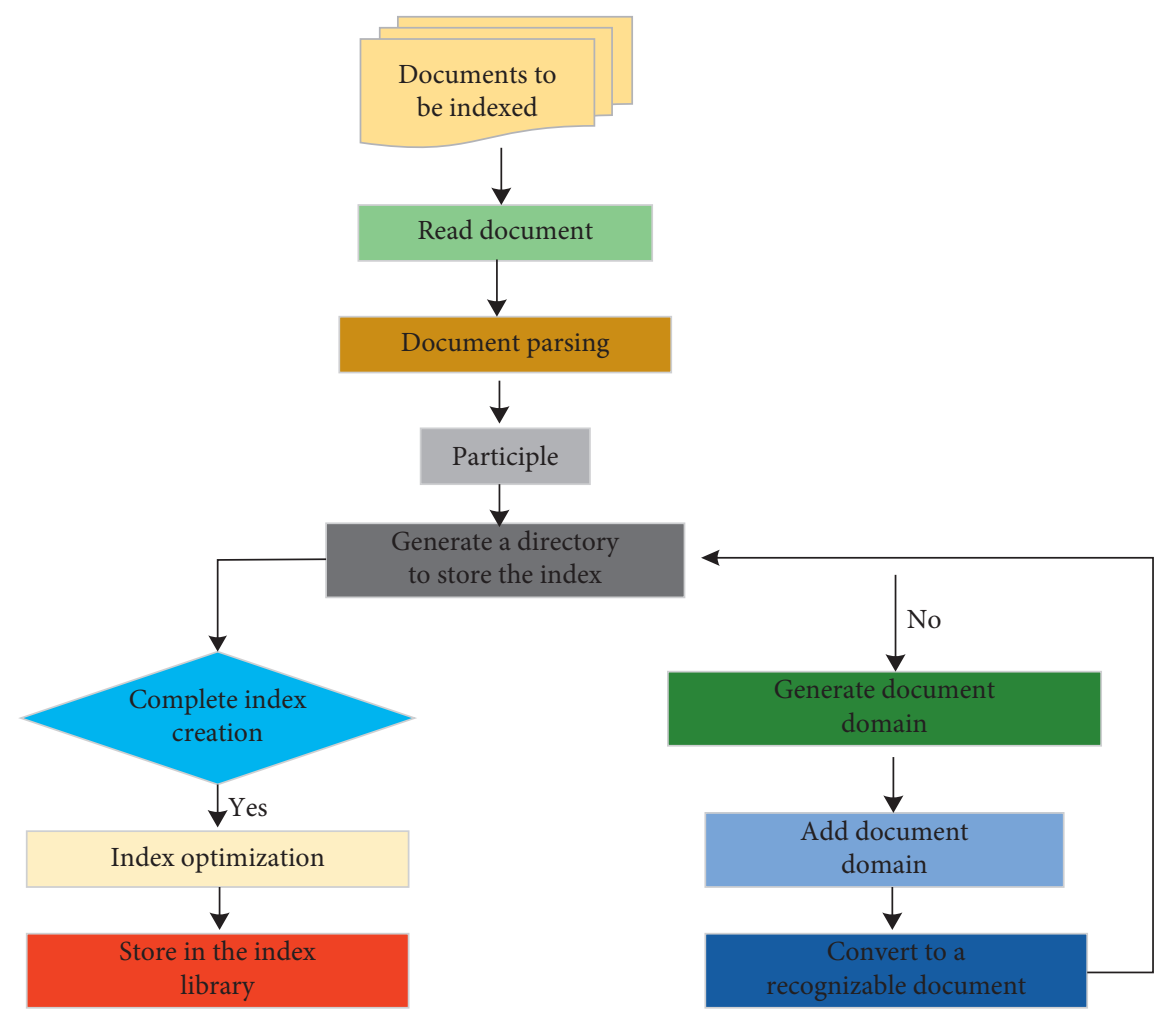

FIgure 4: The process of index creation.

negative samples on the establishment of the category vector is limited, and it may even bring more noise. The classification results are evaluated using the microaverage classification accuracy rate Micro-F and the value evaluation standard. The method of composing training documents is as follows: the training documents of the first-level category are composed of documents in the subcategories below them. For the first-level categories, when training a certain category, the positive example document set is all the documents of that category, and the negative example document set is the sum of the documents of other categories. The second level of the subcategory training method is to compare each subcategory under the same category. For example, for the subcategories of the language category elementary school language, junior high school language, and high school language, when extracting the characteristic words of the elementary school language category, the normal document set is a document of elementary school language, and the negative example document set is the sum of documents of junior high school language and high school language. This improves the training speed, and at the same time, the feature distinction between subcategories is greater. In the test, a top-down classification method is adopted; that is, the document is first classified into a certain category, and then, the document is then classified by each small category under the category to continue to determine whether it belongs to a small category.

4.2. Analysis of Correct Rate Results. The first round of experiments compared six commonly used feature selection methods of DF, IG, Cross Entry, CHI, WET, and MI. Go to the second layer, which is the Micro-F of the subcategory, and the value is shown in Figure 7. It can be seen from the two figures that, in addition to mutual information, other algorithms have achieved better classification results. Among them, the Micro-F of the major category has a very high value, with the maximum value reaching $97.1 \%$. With the decrease in the number of features, the overall trend is declining, but the Micro-F values of various methods are not much different before 3000 dimensions. The Micro-F value of text evidence weight is significantly reduced after $300 \mathrm{D}$ dimensions. Another Micro-F value shows an obvious downward trend after 1000 dimensions. The Micro-F value of the small category is up to $78.2 \%$, which is much lower than the Micro-F of the large category. This may be because the discrimination between the small categories under the same category is relatively poor, resulting in poor classification results, but the overall trend graph is similar to that of major categories.

In feature selection, the function of feature in classification is expressed as the weight to select important features. In the text description, we need to give weight to the features to describe the importance of the text features in the text. In essence, the consideration of feature importance in these two places is the same, which is based on the classification ability and description ability of features. However, the scope of their consideration is different, and the methods adopted are also different. In the face of the whole data set, the importance of features is for all categories, and the distribution of features among categories is emphasized. The latter is for a single text, and the importance of features is for the content 


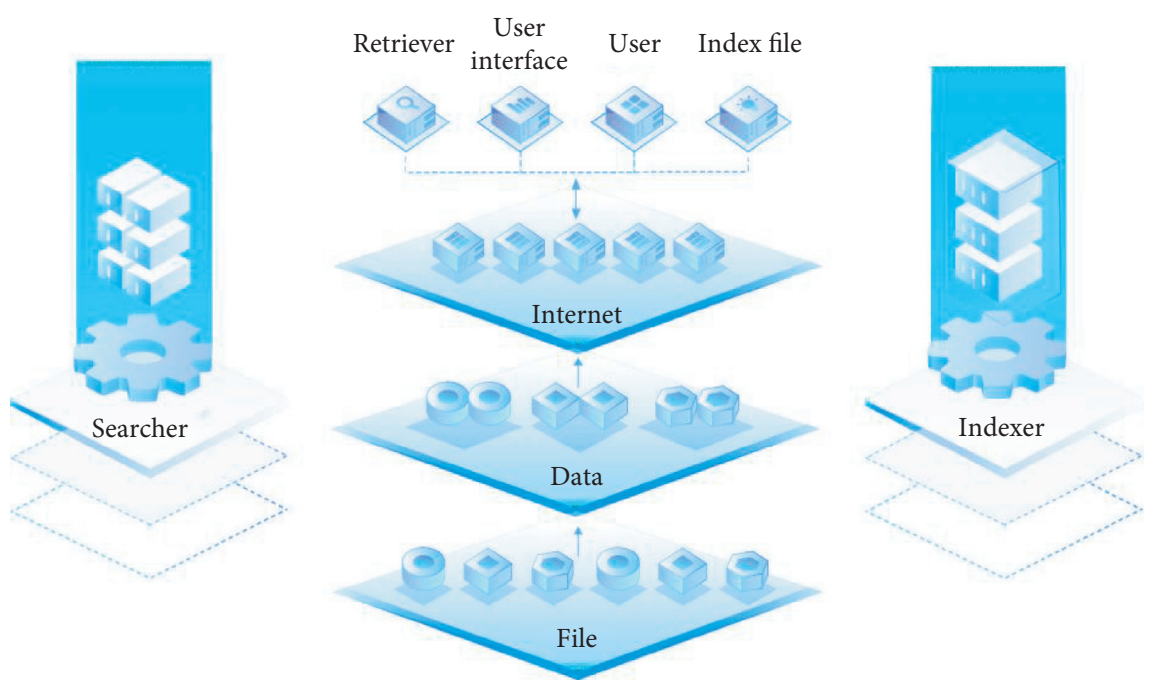

FIgURE 5: The basic process of intelligent information retrieval.

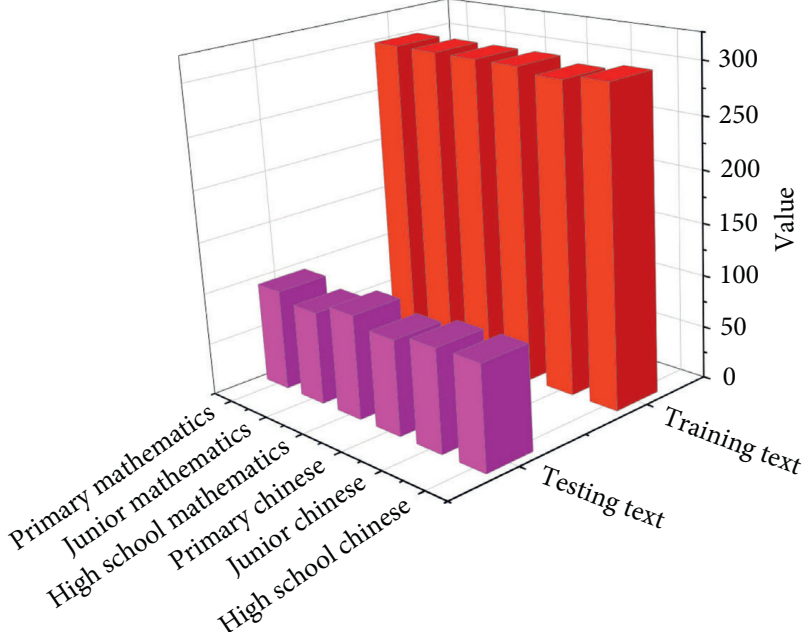

(a)

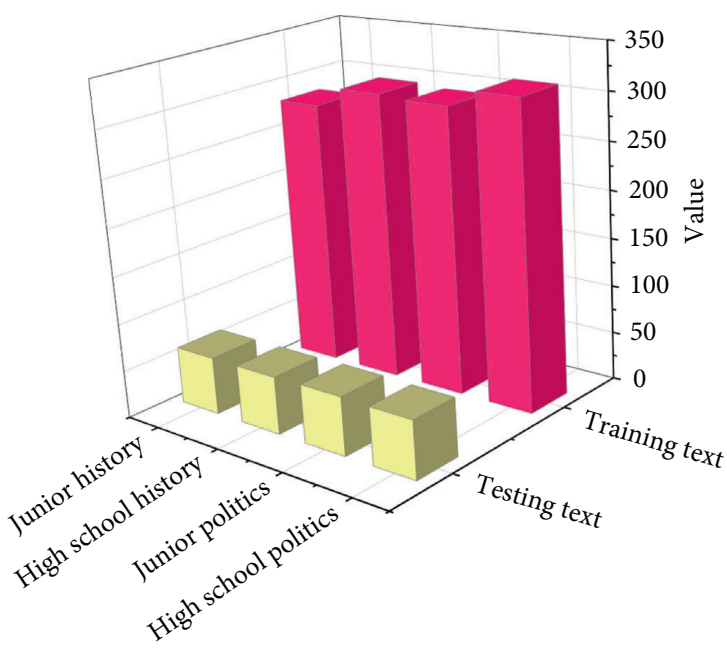

(c)

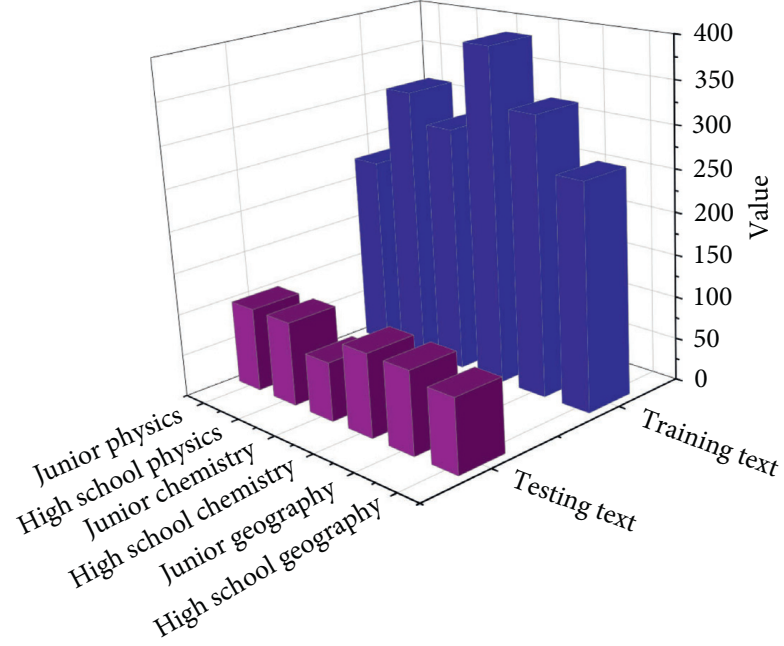

(b)

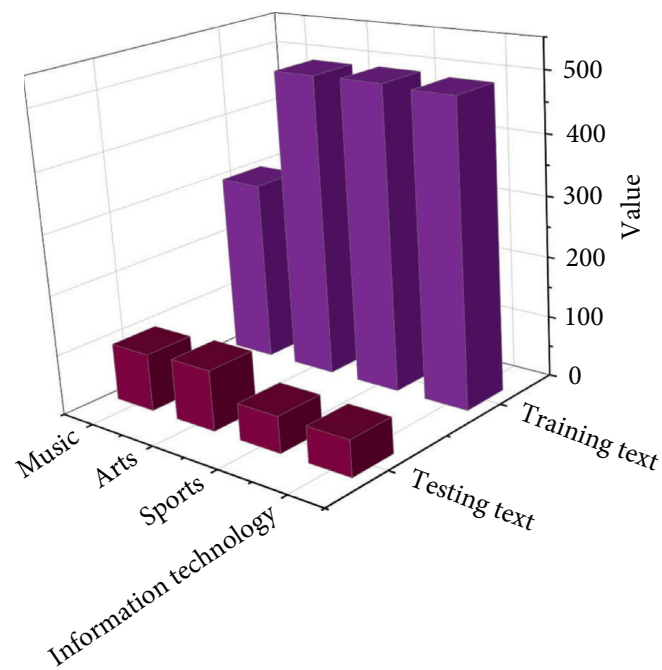

(d)

Figure 6: Distribution table of the training set and test set. 

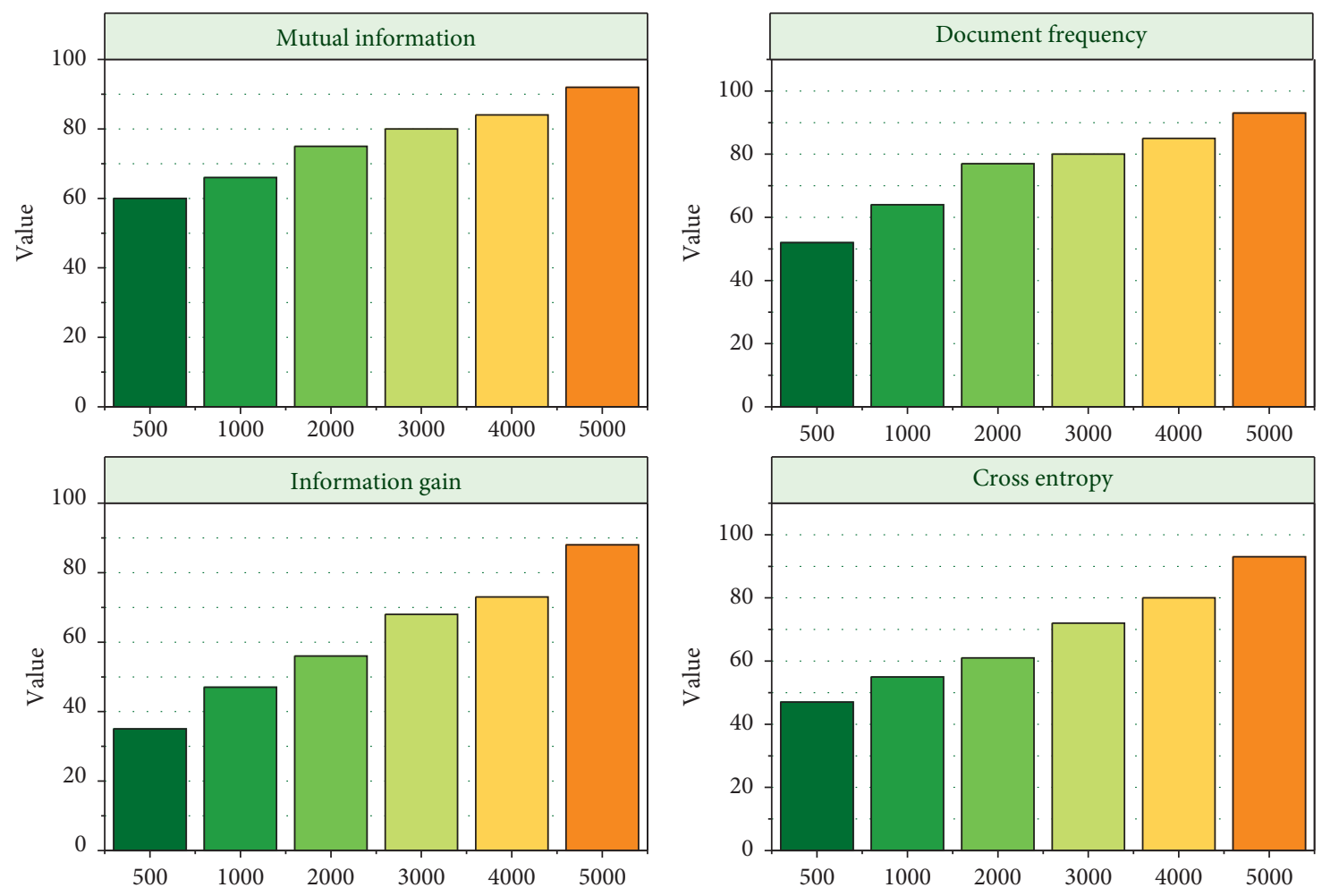

FIgURE 7: The accuracy of the first kind of microaverage classification.

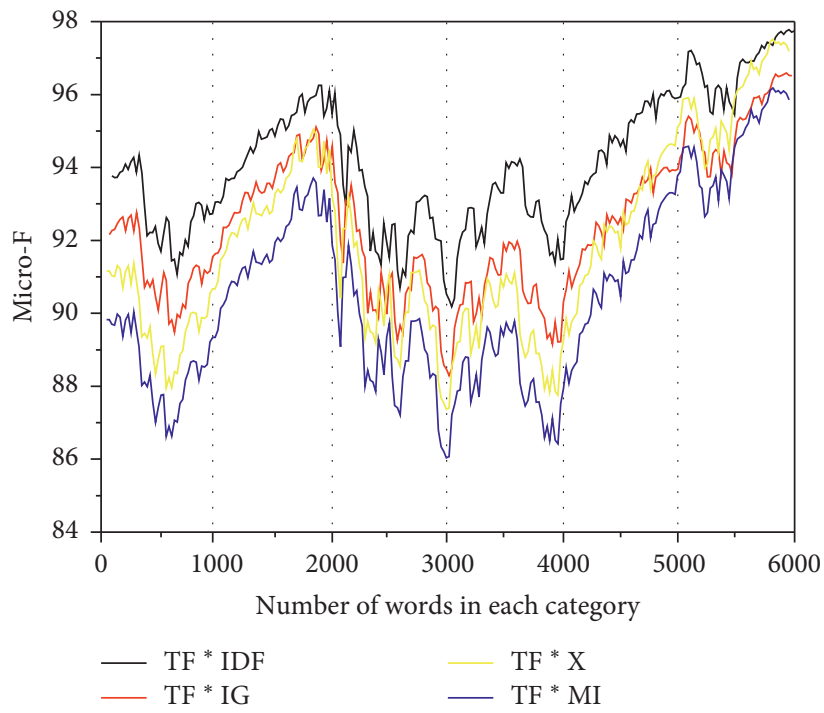

FIGURE 8: Accuracy of microaverage classification.

of the text. In order to measure the description ability and classification ability of features in the text, it also needs to be placed in the global scope to measure accurately.

4.3. Analysis of Classification Results. The Roccio classifier is used to perform weighting experiments on 1867 documents in 12 categories and 18 subcategories of the basic education text set. In this experiment, no dimensionality reduction algorithm is used. The microaverage Micro-F value test is used for the test results. Figure 8 shows the accuracy of the microaverage classification of texts classified into major categories. It can be seen from the figure that TF* IDF has the best classification effect, $\mathrm{TF}^{*}$ Igorot and $\mathrm{TF}^{*} \mathrm{X}$ are slightly worse than $\mathrm{TF}^{*} \mathrm{IDF}$, and $\mathrm{TF}^{*} \mathrm{MI}$ has the worst effect, followed by $\mathrm{TF}^{*} \mathrm{WET}$ and $\mathrm{TF}^{*} \mathrm{MIPW}$. The overall trend of TF* IDF is that as the feature dimension decreases, the classification effect becomes worse, but at 3000 dimensions, 


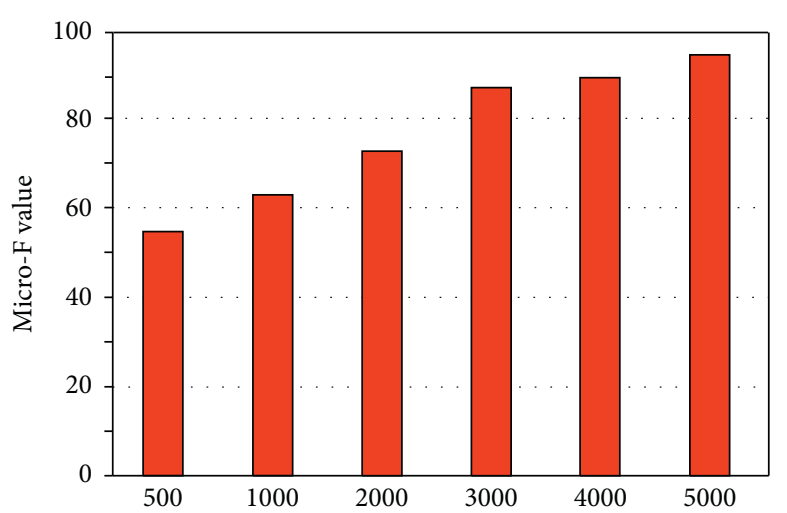

(a)

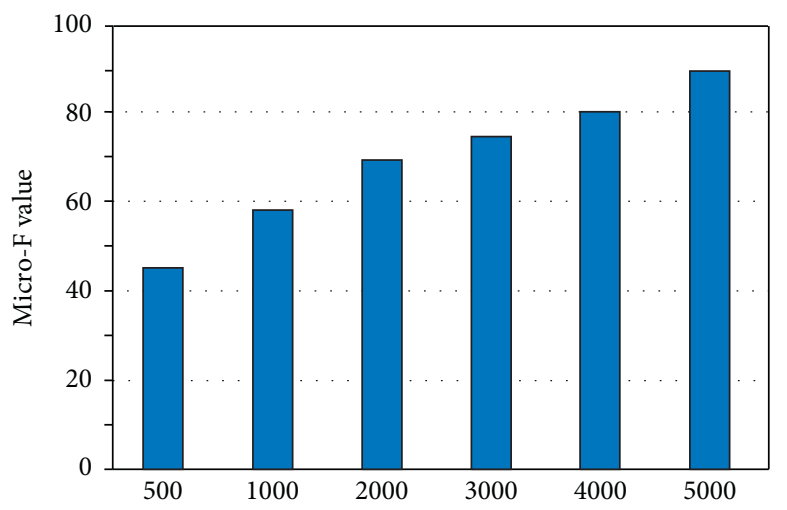

(c)

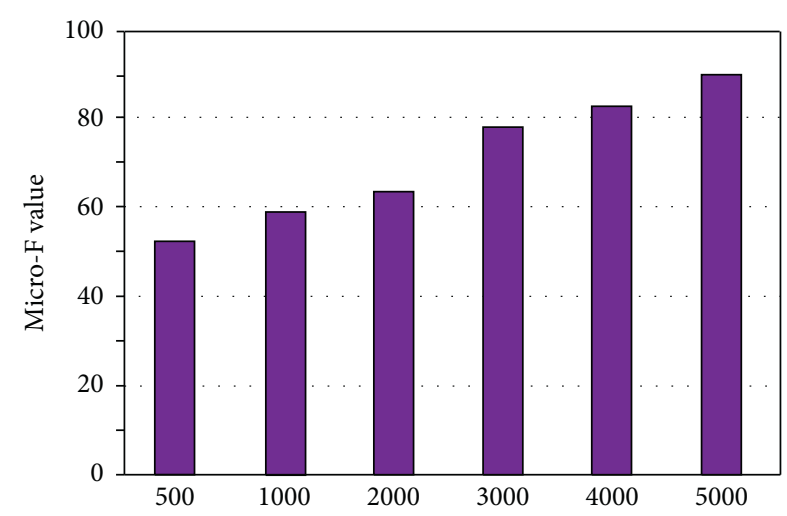

(b)

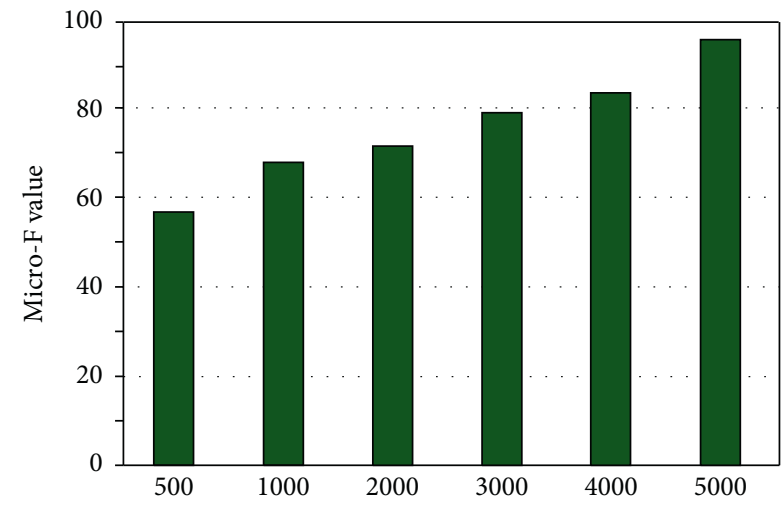

(d)

FIGURE 9: Accuracy of microaverage classification. (a) Classification results of TF $*$ IDF. (b) Classification results of TF $*$ IG. (c) Classification results of $\mathrm{TF} * \mathrm{X}$. (d) Classification results of $\mathrm{TF} * \mathrm{MI}$.

the classification accuracy rate slightly increases. Among them, the Micro-F value is 0.964 .

Figure 9 shows the accuracy of the microaverage classification of texts classified into subcategories. It can be seen from the figure that TF* IDF has the best classification effect, $\mathrm{TF}^{*}$ Igorot, $\mathrm{TF}^{*} \mathrm{X}^{\prime}$, and $\mathrm{TF}^{*} \mathrm{IG}$ are slightly worse than $\mathrm{TF}^{*} \mathrm{IDF}$, and $\mathrm{TF}^{*} \mathrm{MI}$ has the worst effect, followed by TF*WET and TF*MIPW. Except for TF*MI, the overall trend of other functions is that as the feature dimension decreases, the classification effect becomes worse, but the decline is small before 3000 dimensions, and the decline is greater after 3000 dimensions. The Micro-F values of TF* IDF, $\mathrm{TF}^{*}$ Igorot, and $\mathrm{TF}^{*} \mathrm{X}^{2}$ are equivalent at $500 \mathrm{di}^{-}$ mensions. At 3000 dimensions, the Microft value of TF* IDF is 0 . It can be seen that, compared with the classification effect of the large class, the classification accuracy of the small class is still much worse. This may be because, compared with the features of the large class, some small characteristics of the class are not clearly distinguished because of the reasons.

\section{Conclusion}

With the continuous growth of information on the Internet, people have become a way of learning to find what they need through the Internet. In particular, for students in education, their learning is based on resource learning. Therefore, obtaining useful subject knowledge is a key issue for online education students to improve their knowledge. However, due to the limitations of students' knowledge and the control of the Internet, it is difficult to obtain the required knowledge from a general search. Therefore, the development of intelligent search is now increasingly becoming the focus of search research in teaching. With the development of artificial intelligence technology, the intelligence of search engines will become more and more in line with people's actual needs. This article is based on the knowledge in the field of artificial intelligence as the background; according to the characteristics of teaching resources, it is beneficial to search for teaching resources, explore, and build a semantic network in its retrieval module, aiming to classify the domain words in educational resources, so that the retrieval engine can expand the query request based on this concept. This article is to study the use of intelligent search in teaching resources. It is a preliminary exploration of intelligent search engines. Although some methods are proposed, there are still many problems to be studied. This article uses the word frequency method to calculate the degree of correlation between the domain words and the document. It is more practical under certain conditions, but it cannot objectively 
represent the content of the document. Therefore, how to understand and express the content of the document has always been the intelligence of the search engine. The important direction of transformation is not only to be able to extract the main keywords from the document but also to understand the main content of the document, to automatically locate the document accurately, and truly realize the understanding of the conceptual level of the document. With the development of artificial intelligence technology, the key technology of intelligent search will inevitably be greatly improved, and the intelligent search will certainly have broad application prospects in the search field in the future.

\section{Data Availability}

Data sharing is not applicable to this article as no datasets were generated or analyzed during the current study.

\section{Conflicts of Interest}

The authors declare that there are no conflicts of interest.

\section{References}

[1] S. Li and Y. Wang, "Research on interdisciplinary characteristics: a case study in the field of artificial intelligence," IOP Conference Series Materials Science and Engineering, vol. 67, no. 7, pp. 52-53, 2019.

[2] S. Huang, "Design and development of educational robot teaching resources using artificial intelligence technology," International Journal of Emerging Technologies in Learning, vol. 16, no. 5, pp. 116-118, 2021.

[3] I. S. Kang, J. W. Moon, and J. C. Park, "Recent research trends of artificial intelligent machine learning in architectural fieldreview of domestic and international journal papers-," Journal of the Architectural Institute of Korea Structure \& Construction, vol. 33, no. 4, pp. 63-68, 2017.

[4] J. W. Liang and D. B. Wang, "Analysis of research progress in the field of artificial intelligence in the past ten years," Jiangsu Science and Technology Information, vol. 36, no. 11, pp. 22-27, 2019.

[5] Y. Li, "Research on the construction of TCFL resource database system based on artificial intelligence," Journal of Intelligent and Fuzzy Systems, vol. 11, no. 6, pp. 1-12, 2019.

[6] C. Lu, M. J. Guo, F. Y. Zhang et al., "Stakeholder analysis of the application of artificial intelligence in the field of primary health care," Acta Academia Medicine Sinical, vol. 43, no. 1, pp. 101-108, 2021.

[7] H. Liang, "Role of artificial intelligence algorithm for taekwondo teaching effect evaluation model," Journal of Intelligent and Fuzzy Systems, vol. 40, no. 2, pp. 3239-3250, 2021.

[8] L. Feng, J. Wang, C. Ding et al., "Research on the feedback system of face recognition based on artificial intelligence applied to intelligent chip," Journal of Physics: Conference Series, vol. 17, no. 3, pp. 32-34, 2021.

[9] J. Liu, T. Li, J. Chen, and F. Zuo, "Research on improved intelligent control processes based on three kinds of artificial intelligence," Processes, vol. 8, no. 9, pp. 1042-1043, 2020.

[10] G. T. Vu, B. X. Tran, R. S. McIntyre et al., "Modeling the research landscapes of artificial intelligence applications in diabetes (GAPRESEARCH)," International Journal of
Environmental Research and Public Health, vol. 17, no. 6, pp. 1982-1985, 2020.

[11] J. Hu, "Teaching evaluation system by use of machine learning and artificial intelligence methods," International Journal of Emerging Technologies in Learning, vol. 16, no. 5, pp. 87-89, 2021.

[12] A. M. Cox, S. Penfield, and S. Rutter, "The intelligent library: thought leaders' views on the likely impact of artificial intelligence on academic libraries," Library Hi Tech, vol. 37, no. 5, pp. 36-38, 2018.

[13] T. Liang, "Exploration and application of the "Small-Class discussion" teaching mode in the field practice teaching of general geology," Creative Education Studies, vol. 7, no. 5, pp. 623-627, 2019.

[14] Y. Chen, "Research on the development of Chinese intelligent toy industry in the artificial intelligence age," Science Technology and Industry, vol. 17, no. 10, pp. 15-18, 2017.

[15] M. Chen and M. J. Zhang, "Evaluation and research on the design of intelligent classroom teaching in primary school Chinese," Education Teaching Forum, vol. 10, no. 7, pp. 235-239, 2019.

[16] X. Li, “The construction of intelligent English teaching model based on artificial intelligence," International Journal of Emerging Technologies in Learning, vol. 12, no. 2, pp. 35-44, 2017.

[17] B. Hu and B. C. Li, "Applications of artificial intelligence in intelligent manufacturing: a review," Frontiers of Information Technology \& Electronic Engineering, vol. 18, no. 1, pp. 86-96, 2017.

[18] M. Gao, J. Xing, C. Yin et al., "Personalized recommendation method for English teaching resources based on artificial intelligence technology," Journal of Physics Conference Series, vol. 17, no. 1, pp. 12-14, 2021.

[19] B. Garlic, "The application of artificial intelligence in the process of optimizing energy consumption in intelligent areas," Neural Network World, vol. 27, no. 4, pp. 415-446, 2017.

[20] C. Y. Chang, "How the intelligent recognition industry service (IRIS) centre is driving technological research in artificial intelligence," Impact, vol. 20, no. 11, pp. 12-14, 2021.

[21] C. Yang, S. Huan, and Y. Yang, "A practical teaching mode for colleges supported by artificial intelligence," International Journal of Emerging Technologies in Learning (IJET), vol. 15, no. 17, pp. 195-197, 2020.

[22] M. J. Timms, "Letting artificial intelligence in education out of the box: educational cobots and smart classrooms," International Journal of Artificial Intelligence in Education, vol. 26, no. 2, pp. 701-712, 2016.

[23] R. Duan, Y. Wang, and H. Qin, "Artificial intelligence speech recognition model for correcting spoken English teaching," Journal of Intelligent and Fuzzy Systems, vol. 40, no. 1, pp. 1-12, 2020. 